\title{
Diagnóstico territorial de los servicios sociales especializados en menores en la provincia de Castellón
}

\author{
Andrea Badal Camañ \\ al313669@uji.es \\ M. ${ }^{a}$ Raquel Agost-Felip \\ ragost@uji.es
}

\section{Resumen}

La Convención sobre los Derechos del Niño establece la protección del menor por parte del Estado cuando se encuentra en situación de vulnerabilidad. En el actual sistema de servicios sociales, la red de servicios sociales especializados es la encargada de realizar esta función. El presente estudio pretende realizar un diagnóstico territorial de los servicios sociales especializados de atención a la infancia en la provincia de Castellón; este objetivo se plantea dada la escasez de estudios que incorporan la variable territorial en el estudio de los servicios sociales especializados.

Para ello, se ha utilizado una metodología cualitativa de investigación en fuentes secundarias y se han consultado bases de datos oficiales. Las fuentes consultadas son: ARGOS, Mapificación de Centros Inclusivos, el registro de centros acreditados por la Conselleria de Igualdad y Políticas Inclusivas de la Generalitat Valenciana y también webs municipales. Las variables estudiadas son: el ámbito territorial (municipal y comarcal), el tipo de centro, las plazas existentes y la modalidad de gestión (pública y privada).

Los resultados obtenidos en este estudio muestran la escasez de recursos existentes en la provincia de Castellón y su concentración en las comarcas costeras que, a su vez, son las más pobladas. Otro resultado obtenido es que el Servicio de Atención a Familia e Infancia (SEAFI/EEIIA) es el que tiene una mayor presencia territorial.

La infancia y las familias que residen en poblaciones del interior tienen menor acceso al sistema de atención especializado; este patrón de organización territorial se repite en otras provincias. Si se pretende crear un sistema de servicios sociales equitativo que mejore la vida de las familias y la infancia, es necesario reducir estos desequilibrios territoriales en el modelo de atención.

Palabras clave: servicios sociales inclusivos, servicios sociales especializados, infancia, familia, equidad territorial. 


\begin{abstract}
The Convention on the Rights of the Child establishes the protection of children by the state when they are in a situation of vulnerability. In the current system of Social Services, the network of specialized social services is in charge of carrying out this function. The present study aims to perform a territorial diagnosis of the specialized social services for the care of children in the province of Castellón. Therefore, this objective is considered due to the lack of studies that incorporate the territorial variable in the study of the specialized Social Services.

In order to achieve the main goal, a qualitative research methodology has been used in secondary sources, and official databases have been consulted. The sources consulted are: ARGOS, Mapping of Inclusive Centers, the Registry of Centers Accredited by the Department of Equality and inclusive policies of the Generalitat Valenciana, and municipal websites. The variables studied are: territorial scope (municipal and regional), type of center, existing places and management modality. The results obtained in this study show the lack of resources existing in the province of Castellón, and its concentration in the coastal regions which are the most populated. Another result obtained is that the attention service to the family and children (SEAFI/EEIIA) is the one that has a greater territorial presence.

Children and families residing in populations of the interior have less access to the specialized care system, this pattern of territorial organization is the same as in some other provinces. In order to create a system of equitable Social Services which improves the lives of families and children, it is necessary to reduce these territorial imbalances in the model of care.
\end{abstract}

Keywords: inclusive social services, specialized social services, childhood, family, territorial equity.

\title{
Introducción
}

La Unión Europea entiende que algunos de los objetivos principales de los servicios sociales son: 1) proteger contra los riesgos y apoyar a las familias; 2) hacer guardar los derechos humanos; 3) contribuir a la no discriminación reforzando la igualdad; 4) proteger a las personas para mejorar las condiciones de vida y la calidad, garantizando su autonomía y la participación social. Con todo esto, es imprescindible realizar un plan estratégico de servicios sociales en la Comunidad Valenciana, partiendo del análisis de la realidad.

Los derechos y la protección de la infancia y la adolescencia se recogen en diferentes normativas. En el ámbito internacional se encuentra la Convención sobre los Derechos del Niño de Naciones Unidas de 1989 (Ocón Domingo 2003). En el Estado español es la Ley $26 / 2015$, de 28 de julio, de modificación del sistema de protección a la infancia y a la adolescencia, la que proporciona esta protección. En el ámbito autonómico se ha promulgado recientemente la Ley 26/2018, de 21 de diciembre, de la Generalitat Valenciana, de derechos y garantías de la infancia y adolescencia.

Los servicios sociales son un sistema público para la garantía universal de derechos sociales, que tienen como objetivo el bienestar individual para el presente y el futuro. En la actualidad, existe una escasa legislación básica que ordene el sector, lo que da lugar a una desestructuración y dispersión del mismo, si bien se está trabajando en un nuevo modelo social de la Comunidad Valenciana representado, entre otros elementos, por el Plan Valenciano de Inclusión y Cohesión Social (Plan VICS) (Generalitat Valenciana 
2017), Ley 26/2018, de 21 de diciembre, de la Generalitat, de derechos y garantías de la infancia y la adolescencia, y la Ley $3 / 2019$, de 18 de febrero, de servicios sociales inclusivos de la Comunidad Valenciana, aprobadas en los últimos meses. Atendiendo a la legislación en vigor, en el transcurso del desarrollo de este trabajo (Ley 5/1997, de 25 de junio, de la Generalitat Valenciana, por la que se regula el Sistema de Servicios Sociales en el ámbito de la Comunidad Valenciana), los servicios sociales se dividen en dos niveles de atención: generales y especializados.

Los servicios sociales generales constituyen la estructura básica del sistema público de servicios sociales mediante la prestación de una atención integrada y polivalente dirigida a toda la población a través de actuaciones preventivas, asistenciales y rehabilitadoras con ámbito primario, de carácter universal y gratuito. Por otro lado, los servicios sociales especializados van dirigidos a sectores de la población que, por sus condiciones, requieren de un tipo de atención más específica en el terreno técnico y profesional (Ley 5/1997).

Dentro de los servicios sociales especializados se encuentran los dirigidos a menores (véase la figura 1). Para describir los distintos recursos, se hace necesario distinguir entre las diferentes situaciones de desprotección ante las que se pueden encontrar las niñas, los niños y los adolescentes:

1. Situación de riesgo: aquella en la que, a causa de ciertas circunstancias, las niñas, los niños y los adolescentes se vean perjudicados en su desarrollo personal, familiar, social o educativo. En esta situación se interviene desde la Administración pública competente para eliminar, reducir o compensar las dificultades que les afecten y evitar su desamparo y exclusión social. Entre las actuaciones preventivas desarrolladas por los servicios sociales especializados se encuentran:

?] El Servicio Especializado de Atención a Familia e Infancia (SEAFI/EEIIA), formado por un equipo interdisciplinar de ámbito municipal, en este caso la derivación de las intervenciones se realiza desde los servicios sociales generales. El objetivo es la intervención con menores que se encuentren en una situación de riesgo, desamparo o con medidas jurídicas de protección, y con todas las personas que se considere necesario intervenir para conseguir los objetivos pautados. Las actuaciones dentro de la intervención pueden ser individuales, familiares o grupales, y se realizan a través de las técnicas de orientación psicosocial, mediación y psicoterapia, cuya finalidad es la consecución de los objetivos planteados tras la valoración y diagnóstico de la situación.

[? El Punto de Encuentro Familiar (PEF) es un recurso especializado de carácter público y gratuito. Es un espacio neutral donde se reúnen los hijos y las hijas con la madre o el padre no custodio. Es un recurso de ayuda sociopsicopedagògica.

? El centro de día, destinado a atender a niñas, niños y adolescentes durante el día, prestando servicios complementarios de soporte y apoyo familiar. Se clasifican en: centros de día de apoyo convivencial y educativo, donde se realiza una labor preventiva a través de programas de apoyo socioeducativo y familiar, y centros de día de inserción sociolaboral, que realizan una labor preventiva y educativa con adolescentes en situación de riesgo, con el objetivo de potenciar su desarrollo personal y su integración social.

2. Situación de desamparo: se produce a causa del incumplimiento o del imposible ejercicio de los deberes de protección establecidos por las leyes para la guarda de los niños, las niñas y los adolescentes. El acogimiento residencial es una medida de protección que consiste en prestar servicios de alojamiento, manutención, apoyo educativo y atención integral. 


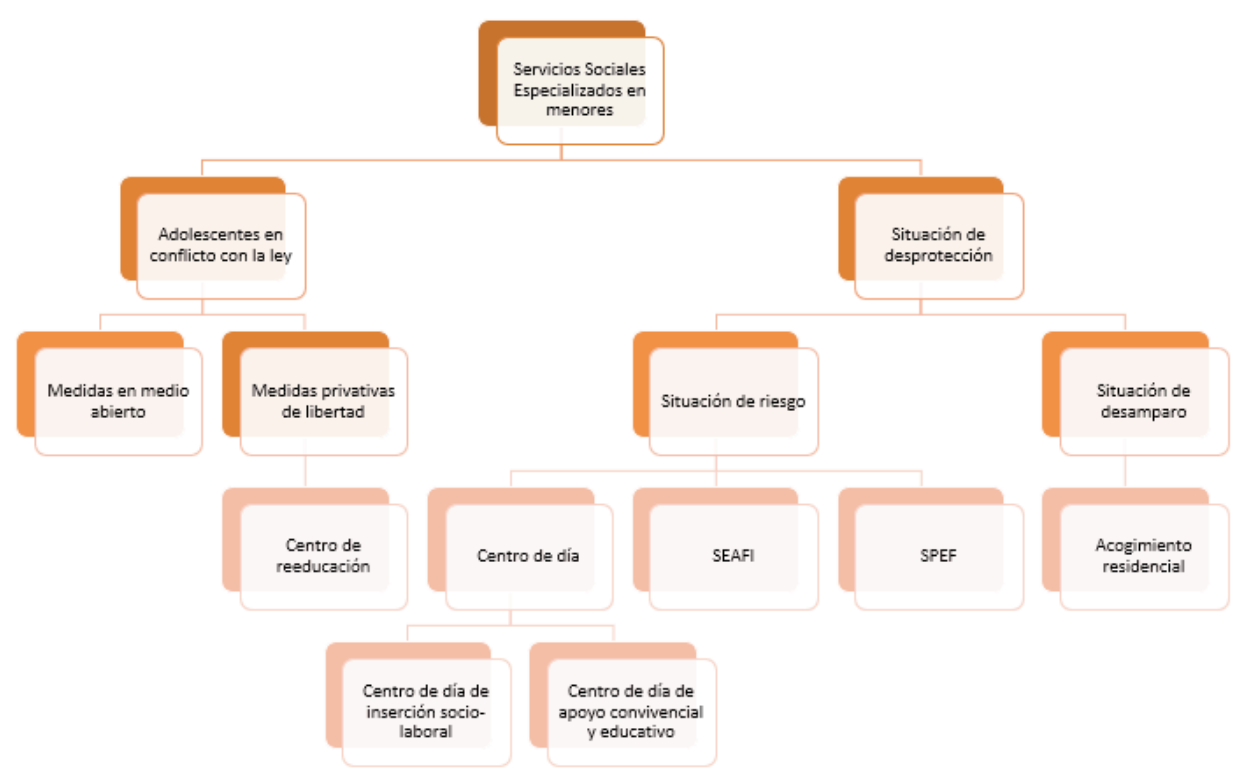

Figura 1. Recursos de los servicios sociales especializados para la infancia y adolescencia.

Además, existen los recursos dirigidos a la atención de la adolescencia en conflicto con la ley, que comprenden las medidas de medio abierto, y los internamientos en centros de reeducación (Ley Orgánica 5/2000).

Tras constatar la escasez de estudios previos sobre servicios sociales especializados en la atención a la infancia y adolescencia diseñados desde una perspectiva territorial en la Comunidad Valenciana, es un tema de estudio incipiente, donde podemos encontrar trabajos como el de Climent López y Uceda-Maza (2017), en el que se realiza una investigación sobre los recursos de servicios sociales especializados en infancia y adolescencia en la provincia de Valencia.

El objetivo del presente trabajo es hacer un diagnóstico territorial de los servicios sociales especializados dirigidos a niñas, niños y adolescentes de la provincia de Castellón. Los objetivos específicos son: a) hacer un diagnóstico territorial de la provincia de Castellón atendiendo al tipo de recurso; $b$ ) hacer un diagnóstico territorial de la provincia de Castellón teniendo en cuenta el municipio y la comarca; $c$ ) conocer el tipo de gestión de los centros (pública o privada); y $d$ ) conocer el número de plazas existentes en los recursos de servicios sociales especializados destinados a la atención de la infancia y la adolescencia.

\section{Metodología}

Para alcanzar los objetivos propuestos, se ha utilizado una metodología cualitativa. El diseño se ha realizado siguiendo el trabajo de Climent López y Uceda-Maza (2017). En dicho trabajo se hace un análisis sobre la red de servicios sociales de la Comunidad Valenciana, haciendo énfasis en los distintos servicios sociales especializados (personas mayores, diversidad funcional, familia e infancia...). En el presente estudio se realiza dicho análisis en el área de familia e infancia, replicando las variables estudiadas en el trabajo de Climent López y Uceda-Maza (2017) y trasladándolo a la provincia de Castellón. Para ello, ha diseñado un análisis descriptivo a partir de fuentes secundarias, en este caso de bases de datos públicas. Las fuentes consultadas han sido tres bases de datos: el portal 
de información ARGOS, ${ }^{1}$ la Mapificación de Centros Inclusivos de la Vicepresidencia y Conselleria de Igualdad y Políticas Inclusivas ${ }^{2}$ y el Registro y Autorización de Funcionamiento de los Servicios y Centros de Acción Social, en la Comunidad Valenciana (Decreto 91/2002). ${ }^{3}$ Esta información se ha completado con las páginas web de los municipios de la provincia de Castellón.

El portal de información ARGOS depende de la Generalitat Valenciana; una de las informaciones que se aporta es el banco de datos municipal, donde se pueden consultar los centros de bienestar social en el municipio que se desea. También se ha consultado la mapificación de centros inclusivos, donde aparecen los centros autorizados por la Conselleria de Igualdad y Políticas Inclusivas, el tipo de gestión y el sector que corresponde. Se ha constatado que la información disponible en estas fuentes no siempre está actualizada, por ello se ha solicitado el registro de centros acreditados de la Conselleria de Igualdad y Políticas Inclusivas en la provincia de Castellón, y se han consultado las páginas web municipales.

Las variables estudiadas son: a) tipo de centro o recurso (SEAFI, centro de día, centro de acogida, centro de inserción sociolaboral); b) el municipio y la comarca donde se encuentran dichos recursos (Alto Mijares, Alto Palancia, Baix Maestrat, Els Ports, Plana Alta, Plana Baixa, l'Alcalatén, Alt Maestrat); c) el tipo de gestión (pública, es decir, municipal y autonómica, o privada, es decir, tercer sector o empresa); d) el número de plazas del centro o recurso.

\section{Resultados}

A continuación, se presentan los resultados para cada uno de los objetivos propuestos. En la tabla 1 se muestra el tipo de centros acreditados que existen en la provincia de Castellón, el tipo de gestión (pública o privada) de los mismos, el número de plazas, así como la distribución territorial según la comarca en la que se encuentran.

A partir de las fuentes consultadas, se han obtenido los siguientes resultados: existen 4 centros de día ( 2 de gestión de la Generalitat Valenciana y 2 de gestión municipal) con 56 plazas, localizados en la Plana Baixa y la Plana Alta. También hay un centro de recepción (gestión de la Generalitat Valenciana) con 30 plazas en la Plana Alta. Hay 11 centros de acogida (4 con gestión de la Generalitat Valenciana y 7 con gestión privada o tercer sector) con un total de 168 plazas; estos centros se ubican en las comarcas de la Plana Alta y la Plana Baixa. También hay un centro de reeducación de la Generalitat Valenciana en la Plana Alta con 47 plazas. Por último, hay 3 puntos de encuentro gestionados por la Generalitat Valenciana distribuidos en las comarcas de la Plana Alta, Plana Baixa y Alt Maestrat.

\footnotetext{
${ }^{1}$ http://www.argos.gva.es/bdmun/pls/argos_mun/DMEDB_UTIL.INDEXC

${ }^{2}$ http://www.inclusio.gva.es/estatico/mapificacion/es/index.html

3 Decreto 91/2002, de 30 de mayo, del Gobierno Valenciano, sobre Registro de los Titulares de Actividades de Acción Social, y de Registro y Autorización de Funcionamiento de los Servicios y Centros de Acción Social, en la Comunidad Valenciana. Obtenido de http://www.dogv.gva.es/datos/2002/06/05/pdf/2002_5931.pdf
} 
Tabla 1.

Distribución de centros, tipo de gestión, número de plazas y comarca.

\begin{tabular}{|c|c|c|c|c|c|}
\hline \multirow[t]{2}{*}{ RECURSOS } & \multicolumn{3}{|c|}{ GESTIÓN } & \multirow[t]{2}{*}{$\begin{array}{l}\text { NÚMERO } \\
\text { PLAZAS }\end{array}$} & \multirow[t]{2}{*}{ COMARCA } \\
\hline & $\begin{array}{l}\text { Generalitat } \\
\text { Valenciana }\end{array}$ & Municipal & $\begin{array}{c}\text { Tercer } \\
\text { sector/ } \\
\text { empresa }\end{array}$ & & \\
\hline Centro de día & 2 & 2 & -- & 56 & $\begin{array}{l}\text { Plana Baixa } \\
\text { Plana Alta }\end{array}$ \\
\hline $\begin{array}{l}\text { Centro } \\
\text { recepción }\end{array}$ & 1 & -- & -- & 30 & Plana Alta \\
\hline Centro de acogida & 4 & -- & 7 & 168 & $\begin{array}{l}\text { Plana Alta } \\
\text { Plana Baixa }\end{array}$ \\
\hline $\begin{array}{l}\text { Centro } \\
\text { reeducación }\end{array}$ & - & -- & 1 & 47 & Plana Alta \\
\hline $\begin{array}{l}\text { Puntos } \\
\text { encuentro }\end{array}$ & 3 & -- & -- & -- & $\begin{array}{l}\text { Plana Alta } \\
\text { Plana Baixa } \\
\text { Alt Maestrat }\end{array}$ \\
\hline
\end{tabular}

A continuación, se puede observar la distribución de manera más visual en los mapas según comarcas (véase la figura 2), donde se puede observar cómo los recursos están distribuidos en la costa y alrededores.
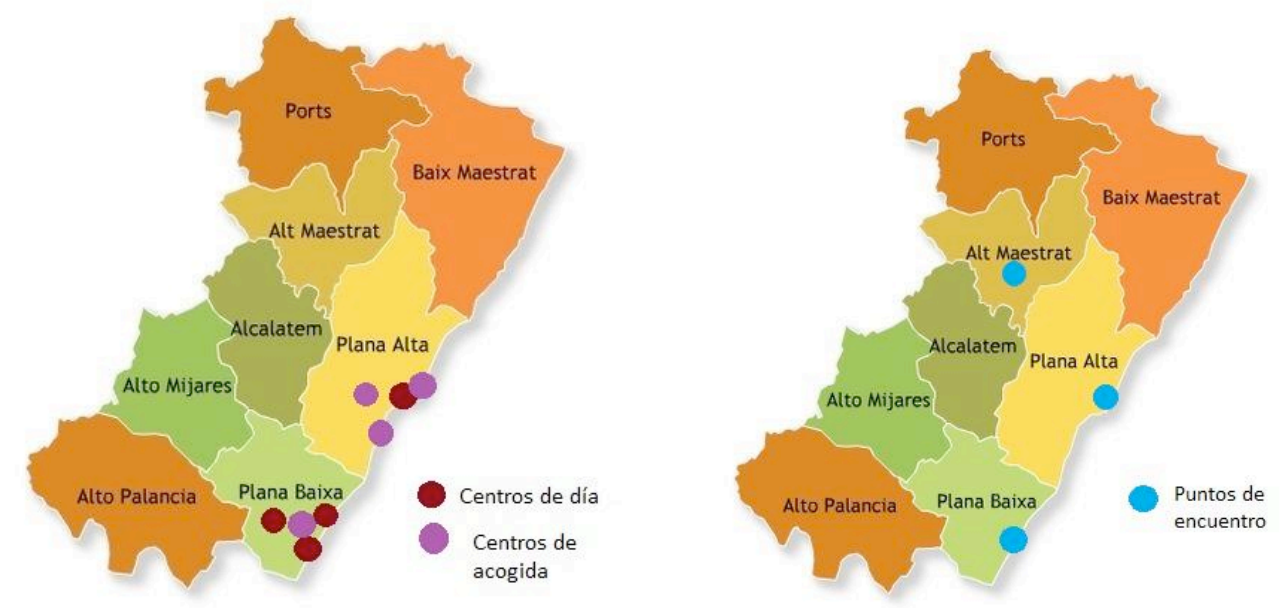

Figura 2. Distribución territorial de centros de día, centros de acogida y puntos de encuentro en la provincia de Castellón en el 2017.

Los SEAFI (en el 2018 se han convertido en equipos específicos de intervención con infancia y adolescencia, EEIIA) son el recurso con el que cuentan más poblaciones, un total de 40 municipios tienen acceso a este servicio en toda la provincia de Castellón, distribuidos en l'Alcalatén, la Plana Baixa, Plana Alta y Baix Maestrat. Y no se encuentra 
este tipo de recursos en las comarcas de Alt Maestrat, Alto Mijares y Els Ports. Todos ellos son de gestión municipal.

\section{Discusión y conclusiones}

Como conclusión, en relación a los tipos de recursos existentes, el SEAFI/EEIIA es el más accesible en parte de los municipios de la provincia, seguido de centros de acogida y centros de día, igual que en la provincia de Valencia (Climent López y Uceda-Maza 2017). En ambas provincias, tanto en Castellón como en Valencia, estos recursos son escasos en el interior. Respecto a la comarca en la que se encuentran los recursos, la red es poco densa, desigual y dispersa; se concentra en núcleos de población y es prácticamente inexistente en otras zonas. Donde más recursos existen es en las comarcas de la Plana Alta y la Plana Baixa, en las comarcas del Alto Mijares y Els Ports no se ha localizado ningún recurso. Respecto al tipo de gestión, lo que más predomina es la gestión municipal y de la Generalitat Valenciana, ya que, como indica la Ley 7/1985 de Bases de Régimen Local, la distribución de competencias debe recaer entre la Administración de la Generalitat y la Administración local. Sin embargo, existen recursos externalizados (gestión del tercer sector y privada). La distribución territorial de los municipios que tienen acceso a un SEAFI/EEIIA sigue el mismo patrón que los otros recursos, concentrándose en zonas costeras y alrededores.

Por último, el número de plazas es escaso en la provincia de Castellón. En comparación con Valencia, que tiene 839 plazas de centros de día (Climent López y Uceda Maza 2017), Castellón tiene 56. Esto se traduce en que el 0,03\% de la población de Valencia podría optar a una plaza en dicho centro, mientras que en Castellón sería un 0,009 \% los que podrían optar a la plaza.

En resumen, la infancia y las familias de las comarcas del interior tienen un acceso reducido al sistema de servicios sociales especializados en menores. Este patrón de organización territorial se repite en otras provincias (Escribano Pizarro, Harráiz Lizan y Serrano Lara 2017). Para crear un sistema de servicios sociales equitativos es necesario reducir estos desequilibrios territoriales.

Una de las limitaciones del estudio es la dificultad de contar con bases de datos actualizadas y centralizadas, dado que, a pesar de las fuentes consultadas, se sigue constatando la dificultad de acceso a la información y la actualización de la información disponible, si bien es cierto que en los últimos meses se viene trabajando en la actualización de la mapificación de los centros inclusivos. Mientras tanto, estudios como este pueden contribuir a conocer la situación de los centros de atención a la infancia y la adolescencia desde una perspectiva territorial, que permita mejorar la planificación de políticas públicas que mejoren la situación y la atención de la infancia y la adolescencia más vulnerable.

\section{Referencias bibliográficas}

Anteproyecto de Ley, de 30 de enero de 2018, de la Generalitat, de Servicios Sociales Inclusivos de la Comunidad Valenciana.

Climent López, Marta y Francesc Xavier Uceda-Maza. 2017. «Los Servicios Sociales para la familia e infancia». En Los Servicios Sociales en la provincia de Valencia: análisis territorial y estado de la cuestión, coord. Lucía Martínez Martínez y Francesc Xavier Uceda-Maza. Valencia: Publicacions de la Universitat de València. 
Escribano Pizarro, Jaime, Cristina Herráiz Lizan y José Javier Serrano Lara. 2017. «La organización territorial de los Servicios Sociales especializados en la provincia de Valencia. Un (des)ajuste entre oferta y demanda». En Los Servicios Sociales en la provincia de Valencia: análisis territorial y estado de la cuestión, coord. Lucía Martínez Martínez y Francesc Xavier Uceda-Maza. Valencia: Publicacions de la Universitat de València.

Generalitat Valenciana. 2017. Plan Valenciano de Inclusión y Cohesión Social (Plan VICS). Recuperado de: http://www.gvaoberta.gva.es/documents/7843050/162503956/PVICS+valencià 2017-05-04.pdf/4a0cdae2-3ab1-4f44-865c-ffe45c913883

Ley $5 / 1997$, de 25 de junio, de la Generalitat Valenciana, por la que se regula el Sistema de Servicios Sociales en el ámbito de la Comunidad Valenciana. Diario Oficial de la Comunidad Valenciana, 12 de agosto de 1997, núm. 192.

Ley $26 / 2018$, de 21 de diciembre, de la Generalitat, de derechos y garantías de la infancia y la adolescencia. Diario Oficial de la Comunidad Valenciana, 24 de diciembre de 2018, núm. 8450.

Ley $3 / 2019$, de 18 de febrero, de la Generalitat, de Servicios Sociales Inclusivos de la Comunidad Valenciana. Diario Oficial de la Comunidad Valenciana, 21 de febrero de 2019, núm. 8491.

Naciones Unidas. 1989. Convención sobre los Derechos del Niño. Ginebra: Organización de las Naciones Unidas.

Ocón Domingo, José. 2003. «Evolución y situación actual de los recursos de protección de menores en España». Revista del Ministerio de Trabajo y Asuntos Sociales 3: 13. 